\title{
Radio study of the extended TeV source VER J1907+062
}

\author{
L. Duvidovich, ${ }^{1 \star}$ A. Petriella, ${ }^{1,2}$ and E. Giacani ${ }^{1,3}$ \\ ${ }^{1}$ CONICET-Universidad de Buenos Aires, Instituto de Astronomía y Física del Espacio (IAFE), Buenos Aires, Argentina \\ ${ }^{2}$ Universidad de Buenos Aires, Ciclo Básico Común, Buenos Aires, Argentina \\ ${ }^{3}$ Universidad de Buenos Aires, Facultad de Arquitectura, Diseño y Urbanismo, Buenos Aires, Argentina
}

Accepted XXX. Received YYY; in original form ZZZ

\begin{abstract}
This paper aims to provide new insights on the origin of the TeV source VER J1907+062 through new high-quality radio observations. We used the Karl G. Jansky Very Large Array (VLA) to observe the whole extension of VER J1907+062 at $1.5 \mathrm{GHz}$ with a mosaicking technique and the PSR J1907+0602 in a single pointing at $6 \mathrm{GHz}$. These data were used together with ${ }^{12} \mathrm{CO}$ and atomic hydrogen observations obtained from public surveys to investigate the interstellar medium in the direction of VER J1907+062. The new radio observations do not show any evidence of a pulsar wind nebula (PWN) driven by the pulsars present in the field and no radio counterpart to the proposed X-ray PWN powered by PSR J1907+0602 is seen in the new VLA image at $6 \mathrm{GHz}$ down to a noise level of $10 \mu \mathrm{Jy} \mathrm{beam}^{-1}$. Molecular clouds were discovered over the eastern, southern, and western borders of the radio shell of G40.5-0.5, suggesting an association with the SNR. We explored several scenarios for the origin of VER J1907+062. We propose as the most probable scenario one in which the TeV emission is produced by two separated $\gamma$-ray sources located at different distances: one of leptonic origin and associated with a PWN powered by PSR J1907+0602 at $\sim 3.2 \mathrm{kpc}$ and another of hadronic origin and produced by the interaction between G40.5-0.5 and the surrounding molecular gas at $~ 8.7 \mathrm{kpc}$.
\end{abstract}

Key words: ISM: individual object: VER J1907+062 - Pulsars: individual object: PSR J1907+0602 - ISM: supernova remnants - ISM: individual object: SNR G40.5-0.5 - ISM: clouds - radio continuum: ISM

\section{INTRODUCTION}

The TeV source VER J1907+062 is an example of the socalled very high energy (VHE) $\gamma$-ray dark sources, which are detected in the VHE range but do not have a clear counterpart at other energy bands. It was first discovered by the MILAGRO Collaboration and identified as the extended VHE source MGRO 1908+06 (Abdo et al. 2007). Aharonian et al. (2009) reported on follow-up observations with H.E.S.S. and discovered some hint of spatial variation of the $\mathrm{TeV}$ spectrum. They found that the emission peaks are slightly offset when the excess events are separated into two energy bands $(0.7-2.5 \mathrm{TeV}$ and $>2.5 \mathrm{TeV})$, being the high-energy emission harder than the low energy one. However, the systematic errors forbid any conclusion on the separation in two sources or peaks. In the VHE regime, the region was also observed with VERITAS between 2007 and 2012 (designated as VER J1907+062), totalizing about $62 \mathrm{hr}$ of useful exposure (Aliu et al. 2014). The new $\gamma$-ray excess map shows strong $\mathrm{TeV}$ emission near the location of the pulsar PSR

^ E-mail: lduvidovich@iafe.uba.ar
J1907+0602, although the peak of the excess counts is offset to the west. As first observed by H.E.S.S., the TeV emission also extends toward the SNR G40.5-0.5 (see Fig. 1).

The $\gamma$-ray pulsar PSR J1907+0602 was first discovered by Abdo et al. (2009) using the Fermi-LAT telescope. It has a characteristic age of $19.5 \mathrm{kyr}$ and a spin-down luminosity of $3 \times 10^{36} \mathrm{erg} \mathrm{s}^{-1}$ (Abdo et al. 2010). These authors report on the detection of pulsed emission in the radio band using the Arecibo $305 \mathrm{~m}$ telescope. The timeaveraged flux density at $1.4 \mathrm{GHz}$ is $3.4 \mu \mathrm{Jy}$ and the dispersion measure (DM) of $82.1 \mathrm{pc} \mathrm{cm}^{-3}$ implies a distance of $3.2 \pm 0.6 \mathrm{kpc}$, using the model for the electron distribution of Cordes \& Lazio (2002). The authors also analyze the emission of PSR J1907+0602 in the X-ray band using Chandra observations. They find a point source with a hard non-thermal spectrum and some excess of diffuse emission around it, which could originate in a compact pulsar wind nebula (PWN) powered by the pulsar. XMM-Newton observations also show that PSR J1907+0602 is a point source with marginal excess X-ray emission, interpreted as a bow shock in front of the pulsar moving through the interstellar medium (ISM) (Pandel \& Scott 2012). Pandel (2015) 
reported on the non detection of diffuse X-ray emission associated either with VER J1907+062 or with the SNR G40.5-0.5.

The SNR G40.5-0.5 presents in the radio band a nonthermal shell of $22^{\prime}$ in diameter and a spectral index $\alpha \sim 0.4$ $\left(S_{v} \propto \alpha^{-v}\right)$ (Sun et al. 2011). In the radio continuum image at $1.4 \mathrm{GHz}$ of the Very Large Array Galactic Plane Survey (VGPS, Stil et al. 2006), the shell appears well defined in the NE direction and more diffuse toward the SW. Using the $\Sigma-D$ relation, where $\Sigma$ is the surface brightness and $D$ the diameter of the SNR, Downes et al. (1980) estimated a distance between 5.5 and $8.5 \mathrm{kpc}$ and an age between 20 and $40 \mathrm{kyr}$. Recently, the Pulsar Arecibo L-Band Feed Array (PALFA) project confirmed the presence of the pulsar PSR J1907+0631 lying near the center of G40.5-0.5 (Lyne et al. 2017) and the authors suggested an association between them. It is a young pulsar $\left(\tau_{c} \sim 11 \mathrm{kyr}\right)$ with a spindown luminosity of $\sim 5 \times 10^{35} \mathrm{erg} \mathrm{s}^{-1}$ and a DM of $\sim 430 \mathrm{pc}$ $\mathrm{cm}^{-3}$, which translates into a distance of $7.9 \mathrm{kpc}$, using also the model for the electron distribution of Cordes \& Lazio (2002). The authors also report on the presence of a region of enhanced radio emission at the position of PSR J1907+0631, which they interpret as a probable PWN powered by the pulsar.

A third pulsar, PSR J1905+0600, is detected in the direction of VER J1907+062. It is a low energy $\left(\dot{E} \sim 5.3 \times 10^{32}\right.$ $\left.\mathrm{erg} \mathrm{s}^{-1}\right)$ and old $(\sim 6 \mathrm{Myr})$ radio pulsar with a high DM of $\sim 730 \mathrm{pc} \mathrm{cm}^{-3}$. This translates into a distance of $18 \mathrm{kpc}$, which places the pulsar outside the solar circle (Hobbs et al. 2004).

So far, the nature of VER J1907+062 has not been determined. Based on the strong $\mathrm{TeV}$ emission around PSR J1907+0602, which in turn presents marginal evidence of being powering a PWN in X-rays, VER J1907+062 was classified as a TeV PWN candidate (Aliu et al. 2014). The luminosity of the pulsar gives a $\mathrm{TeV} \gamma$-ray efficiency similar to that of $\mathrm{TeV}$ PWNe with pulsars of similar spin-down energy (Abdo et al. 2009). However, its large spatial extent (larger than the rest of $\mathrm{TeV}$ PWNe of similar age) and the lack of variation of the $\gamma$-ray spectrum across the TeV emission argue against interpreting it as solely powered by PSR J1907+0602. Moreover, a two-dimensional Gaussian fails to accurately fit the VHE morphology of the source, which indicates that VER J1907+062 may be the superposition of two sources, either separated or interacting. On the other hand, based on the fact that the $\mathrm{TeV}$ emission partially overlaps the SNR G40.5-0.5, Aharonian et al. (2009) first suggested that the interaction between the remnant and the ISM could contribute as an additional source of $\gamma$-rays.

In this paper we report on high-quality radio observations carried out with the Karl G. Jansky Very Large Array ${ }^{1}$ (VLA) toward VER J1907+062 with the aim to investigate different scenarios about its nature. In particular, our purpose is to detect the radio counterpart of the proposed X-ray PWN driven by PSR J1907+0602, as well as explore the rol of the other two pulsars present in the observed field as possible sources of $\mathrm{TeV}$ emission. In addition, we analyze the

1 The Very Large Array of the National Radio Astronomy Observatory is a facility of the National Science Foundation operated under cooperative agreement by Associated Universities, Inc.
Table 1. Summary of VLA observations.

\begin{tabular}{ccc}
\hline \hline & VER J1907+062 & PSR J1907+0602 \\
\hline Configuration & $\mathrm{D}$ & $\mathrm{D}$ \\
Band & $\mathrm{L}$ & $\mathrm{C}$ \\
Date & 2018 Sep 3 & 2018 Sep 2 \\
Integration time & 128 min & 66 min \\
\hline
\end{tabular}

molecular gas in the surroundings of the SNR G40.5-0.5 to determine the possibility that the remnant contributes to the TeV emission from VER J1907+062 via pion decay from proton-proton collisions. In this context, it is expected a correlation between the $\gamma$-ray emission and matter concentration.

\section{OBSERVATIONS AND DATA REDUCTION}

\subsection{New radio observations}

The radio continuum observations toward the whole extension of VER J1907+062 were performed using the VLA in its D configuration (project ID 18A-093). To cover the extension of this large VHE source $\left(\sim 62^{\prime}\right)$, we used a mosaicking technique with 12 different pointings following an hexagonal pattern. The data were taking at L-band using the wide-band $1 \mathrm{GHz}$ receiver system centered at $1.5 \mathrm{GHz}$ which consists of 16 spectral windows with a bandwidth of $64 \mathrm{MHz}$ each, spread into 64 channels. The source 3C 48 was used as primary flux density and bandpass calibrations, while the phase was calibrated with $\mathrm{J} 1859+1259$.

Also, we carried out observations toward the PSR J1907+0602 in a single pointing using the VLA in its D configuration in C-band. These observations were planned to look for the radio PWN around the pulsar, which presents a hint of a PWN in X-ray. In this case, we used the wide-band 4-8 GHz receiver system. We requested the 3 -bit sampler with 2 sub-bands of $2 \mathrm{GHz}$, each comprising 16 spectral windows with a bandwidth of $128 \mathrm{MHz}$ each, spread into 64 channels. The source 3C 48 was used as flux density and bandpass calibrator, and J1922+1530 as a phase calibrator. Table 1 summarizes the observations.

The data at both frequencies were processed through the VLA CASA Calibration Pipeline. We improved the quality of the calibrated data by applying extra flagging before imaging. Cleaned images were obtained with the TCLEAN task of CASA, using the multi-frequency deconvolution algorithm. We applied a Briggs weighting with a robustness parameter of 1.0 and 0.0 for the VER J1907+062 and PSR J1907+0602 images, respectively. At $1.5 \mathrm{GHz}$ the radio image has a synthesized beam of $51^{\prime \prime} .1 \times 39^{\prime \prime} .5$ at a position angle of $21^{\circ} .9$, and a rms noise of $1 \mathrm{mJy}^{\text {beam }}{ }^{-1}$. The final image at $6 \mathrm{GHz}$ has a resolution of $10^{\prime \prime} .2 \times 8^{\prime \prime} .6$ at a position angle of $-48^{\circ} .5$, and a rms noise of $10 \mu \mathrm{Jy}$ beam $^{-1}$.

\subsection{The surrounding medium}

The properties of the ISM around SNR G40.5-0.5 were investigated using the neutral hydrogen (HI) data extracted from the Very Large Array Galactic Plane Survey (VGPS, Stil et al. 2006), which maps the HI $21 \mathrm{~cm}$ line emission 


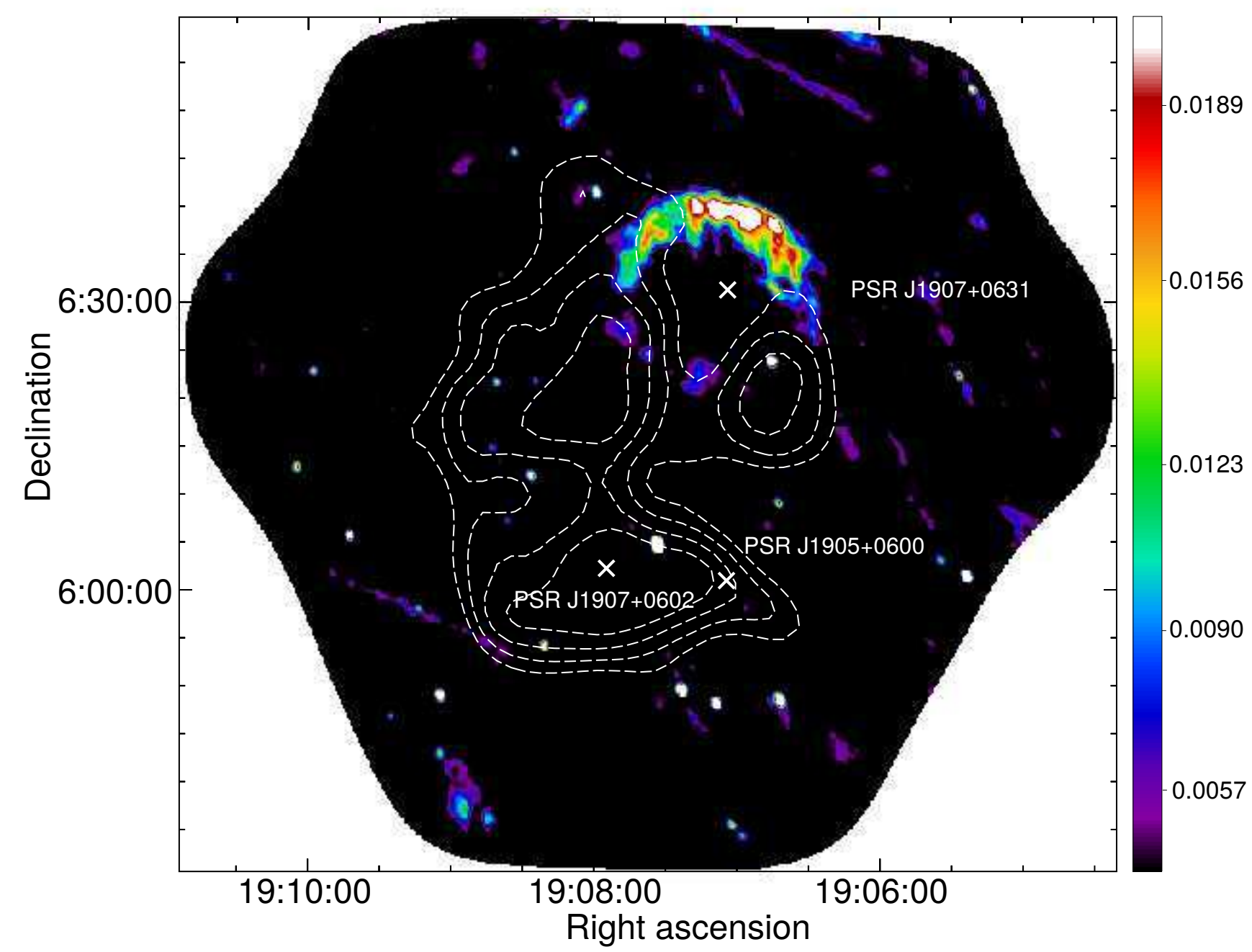

Figure 1. Radio continuum image at $1.5 \mathrm{GHz}$ covering the whole extension of the TeV source VER J1907+062. This image was obtained from a combination of 12 different pointings observed with the VLA in the D configuration. The angular resolution is $51^{\prime \prime} .1 \times 39^{\prime \prime} .5$, PA $=21^{\circ} .9$ and the rms noise is $1 \mathrm{mJy}_{\text {beam }}^{-1}$. The color scale to the right of the image is expressed in Jy beam ${ }^{-1}$. The cross signs indicate the position of the pulsars PSR J1907+0631, PSR J1907+0602, and PSR J1905+0600. The dotted contours represent the TeV emission from VERITAS extracted from Aliu et al. (2014).

with angular and spectral resolutions of $1^{\prime}$ and $1.3 \mathrm{~km} \mathrm{~s}^{-1}$, respectively. Molecular line emission was extracted from the FOREST Unbiased Galactic Plane Imaging ${ }^{2}$ (FUGIN) survey, which maps the 1st and 3rd Galactic quadrants in the ${ }^{12} \mathrm{CO}(\mathrm{J}=1-0)$ using the multi-beam FOREST receiver installed on the Nobeyama 45-m telescope (Umemoto et al. 2017). The angular and velocity resolutions are $20^{\prime \prime}$ and 0.65 $\mathrm{km} \mathrm{s}^{-1}$, respectively.

\section{RESULTS}

\subsection{The new VLA images}

The new VLA image at $1.5 \mathrm{GHz}$ containing the whole extension of the TeV source VER J1907+062 is shown in Fig. 1. A

\footnotetext{
2 Retrieved from the JVO portal (http://jvo.nao.ac.jp/portal/) operated by ADC/NAOJ.
}

number of compact sources can be seen in the field together with the SNR G40.5-0.5. A spurious instrumental artifact visible toward the $\mathrm{N}$ of the field is due to the presence of the bright HII region G041.096-00.213 that lies outside the observed area.

The new radio data delineate the shell of the southern hemisphere of the remnant, almost lost in the continuum image of the VGPS, thus completing a circular shell with the northern hemisphere much brighter. The recently detected PSR J1906+0631 lies in projection of the sky close to the center of the remnant. The enhanced radio emission at the position of the pulsar, proposed to be a probable PWN by Lyne et al. (2017), is not detected with our deeper observations. We suggest that this feature, which is seen in the VGPS data, could be caused by an interference effect produced by the strong point source located at R.A. $=19^{\mathrm{h}} 06^{\mathrm{m}} 45^{\mathrm{s}} .7$, Dec. $=6^{\circ} 23^{\prime} 50^{\prime \prime}(\mathrm{J} 2000)$. On the other hand, the new radio image does not show any evidence of extended 
emission in coincidence with PSR J1907+0602 down to the noise level of our observations.

Fig. 2 displays the new VLA radio image at $6 \mathrm{GHz}$ centered at the position of PSR J1907+0602 (R.A. = $19^{\mathrm{h}} 07^{\mathrm{m}} 54^{\mathrm{s}} .73$, Dec. $\left.=6^{\circ} 02^{\prime} 16^{\prime \prime} .9(\mathrm{~J} 2000)\right)$. Also in this case, our new high-sensitivity radio observations show no evidence of extended nor point-like emission toward the pulsar. This result does not allow us to dismiss the suggestion made by Pandel \& Scott (2012) that the non-thermal X-ray emission around the pulsar may be a PWN. There are numerous examples of confirmed X-ray PWN with no detected counterpart in the radio band (see, for example, the SNRcat $^{3}$ of Ferrand \& Safi-Harb 2012).

Several deep sensitivity and high resolution radio studies searching for radio PWNe were performed with negative results (e.g., Gaensler et al. 2000; Giacani et al. 2014; Castelletti et al. 2016; Sushch et al. 2017). In these cases, the lack of detectable radio emission has been explained by the presence of either pulsars with high magnetic field that inhibit the production of synchrotron radiation at longer wavelengths, or the presence of young and energetic pulsars residing in very low ambient density media $(\sim 0.003$ $\mathrm{cm}^{-3}$ ), which results in severe adiabatic losses and consequently under-luminous radio PWNe. Neither of these scenarios seem to apply for PSR J1907+0602. On one hand, its magnetic field is about $3 \times 10^{12} \mathrm{G}$, consistent with most of young pulsars associated with SNRs (Gaensler \& Slane 2006). On the other hand, to investigate the density in the environs of PSR J1907+0602, first we converted from the pulsar's distance of $3.2 \mathrm{kpc}$ to a velocity of $\sim 57 \mathrm{~km} \mathrm{~s}^{-1}$, using the universal rotation curve of Persic et al. (1996) with the parameters of Reid et al. (2014) (Galacto-centric distance $R_{0}=8.34 \mathrm{kpc}$ and rotation velocity of the Sun $\Theta=241 \mathrm{~km}$ $\mathrm{s}^{-1}$ ) (all velocities hereafter are referred to the local standard of rest). To estimate the atomic density of the ISM where the pulsar lies, we must take into account that the DM does not precisely matches the kinematical distance, i.e. there are errors involved when converting from $\mathrm{DM} \sim 82$ pc $\mathrm{cm}^{-3}$ to a distance of $\sim 3.2 \mathrm{kpc}$ and hence to the kinematical velocity of $\sim 57 \mathrm{~km} \mathrm{~s}^{-1}$. In order to consider such uncertainties, we analyzed the atomic gas distribution in the velocity interval $57 \pm 10 \mathrm{~km} \mathrm{~s}^{-1}$ and performed 4 integration of the HI emission into velocity intervals of $\sim 4 \mathrm{~km} \mathrm{~s}^{-1}$, namely $\sim[47.2-51.3], \sim[52.1-56.2], \sim[57.1-61.2]$ and $\sim[62.0-67.0] \mathrm{km} \mathrm{s}^{-1}$. We estimated the HI column density $N_{H I}\left[\mathrm{~cm}^{-2}\right]=1.82 \times 10^{18} \int T_{B} d v$, where $T_{B}$ is the brightness temperature in $\mathrm{K}$ and the integration was performed in the aforementioned intervals. We assume that the emission comes from spherical regions with a radius of $\sim 0^{\circ} .2$ located at distances of $\sim 2.8,3.1,3.4$ and $3.7 \mathrm{kpc}$, as derived from the central velocity of each integration interval. We obtained densities between $\sim 5 \mathrm{~cm}^{-3}$ to $\sim 15 \mathrm{~cm}^{-3}$. Even after considering the uncertainties in the densities, which can be $\sim 40 \%$ and are caused by the error in distance, the choice of the background level and in the integration boundaries, the obtained values rule out the possibility of an expansion in an area of very low density.

As proposed by Frail \& Scharringhausen (1997) and

3 Current catalog available at

http://www.physics.umanitoba.ca/snr/SNRcat/

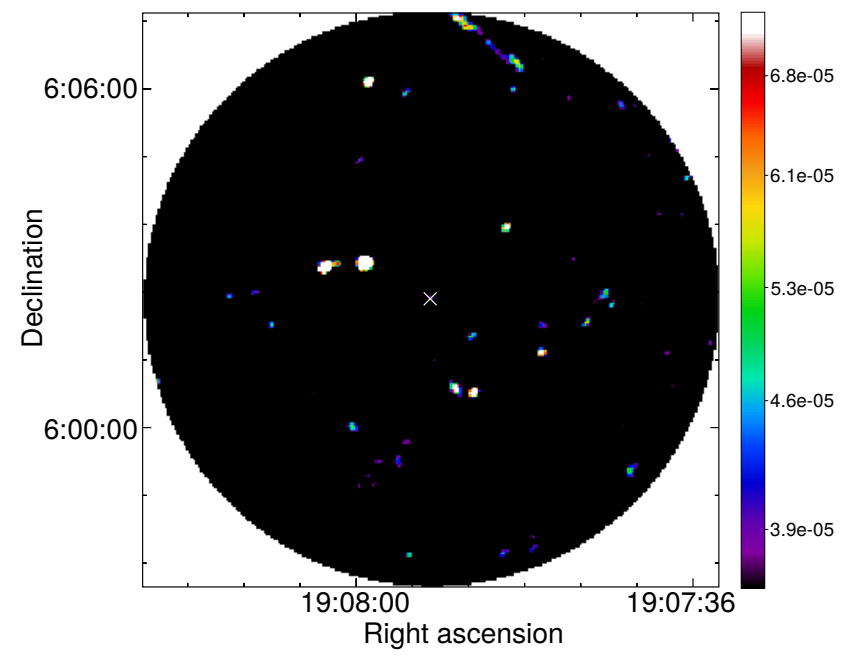

Figure 2. Radio continuum image at $6 \mathrm{GHz}$ of the region surrounding the position of the pulsar PSR J1907+0602 (marked with a cross) and constructed using the VLA in its D array. The final beam size, is $12^{\prime \prime} .2 \times 8^{\prime \prime} .6$ while the rms noise level is $10 \mu \mathrm{Jy}$ beam $^{-1}$. The color scale is expressed in Jy beam ${ }^{-1}$.

Gaensler et al. (2000) these pulsars probably would be less efficient in producing radio nebulae because of the injection spectrum of particles in the pulsar wind has shifted to higher energies. Regarding the other pulsar, PSR J1907+0631, its high surface magnetic field of $1.2 \times 10^{13} \mathrm{G}$ could inhibit the production of synchrotron radiation in the radio band, as in the case of the $\gamma$-ray pulsar RX J0007.0+7303 (Giacani et al. 2014).

\subsection{The molecular environment of G40.5-0.5}

The determination of the distance to the SNR G40.5-0.5 is important to analyze the connection with the $\mathrm{TeV}$ source. The only estimate of the distance for this remnant was based on the $\Sigma-D$ relationship, which is a method known to have large intrinsic dispersion. A more accurate method for establish distance constraints to radio continuum sources (including SNRs) is based on the construction of an absorption HI spectrum. This method has given reliable distance estimates in bright SNRs (see, for example, Ranasinghe \& Leahy 2017). For faint sources, the fluctuations in the HI distribution can give false absorption features whose signals are similar to the true absorption (Ranasinghe \& Leahy 2018).

We constructed an HI absorption spectrum of G40.5-0.5 but did not obtained an acceptable spectrum to constraint the source distance. Although this is a bright SNR, at least over its northern shell, the HI absorption spectrum resulted very noisy, probably due to the fact that the neutral gas is patchy and caused spurious absorption features.

We also analyzed the distribution of the $\mathrm{CO}$ gas in the environment of G40.5-0.5 in order to identify molecular material that shows spatial correlation with both the remnant and the $\gamma$-ray emission. If an association between the SNR and molecular features is established, it serves to better constraint its distance and to explore a hadronic mechanism as a probable origin for the $\mathrm{TeV}$ emission. After a careful in- 
spection of the whole ${ }^{12} \mathrm{CO}(\mathrm{J}=1-0)$ data cube, we found morphological signatures of a possible association in the velocity range between $+56 \mathrm{~km} \mathrm{~s}^{-1}$ and $+75 \mathrm{~km} \mathrm{~s}^{-1}$.

Fig. 3 displays the ${ }^{12} \mathrm{CO}$ distribution integrated over 5 consecutive channels, giving a velocity coverage of $\sim 2.6$ $\mathrm{km} \mathrm{s}^{-1}$ for each map. The red contours delineate the radio continuum emission of the remnant at $1.5 \mathrm{GHz}$, while the yellow contours correspond to the $\mathrm{TeV}$ source. From this figure, we can discern several condensations of molecular gas labeled A, B, and C, bordering the edges S, E and W of G40.5-0.5, respectively. Feature A, observed in the $\sim 56-65$ $\mathrm{km} \mathrm{s}^{-1}$ velocity range (panels $b, c$, and $d$ ) is elongated in the NE-SW direction, and partially overlaps the southern border of the remnant and the NW maximum of the $\mathrm{TeV}$ source. Feature B is seen in the $\sim 66-69 \mathrm{~km} \mathrm{~s}^{-1}$ range (panel e) and projected toward the eastern border of G40.5-0.5. It partially overlaps the NE peak of the $\gamma$-ray emission. Finally, in the $\sim 69-75 \mathrm{~km} \mathrm{~s}^{-1}$ range (panels $f$ and $g$ ), the molecular feature $\mathrm{C}$ appears overlapping the western edge of the radio shell and part of the NW maximum of the TeV emission.

We adopt $\sim 60 \mathrm{~km} \mathrm{~s}^{-1}, \sim 67 \mathrm{~km} \mathrm{~s}^{-1}$, and $72 \mathrm{~km} \mathrm{~s}^{-1}$ as the systemic velocities of molecular features $\mathrm{A}, \mathrm{B}$, and $\mathrm{C}$, respectively. To convert from radial velocities to distances, we use again the universal rotation curve of Persic et al. (1996) with the parameters of Reid et al. (2014). The first Galactic quadrant presents distance ambiguity for positive radial velocities, so we obtain near and far distances of 3.4 and 9.1 $\mathrm{kpc}$ for feature A, 3.9 and $8.6 \mathrm{kpc}$ for feature B, and 4.2 and $8.3 \mathrm{kpc}$ for feature $\mathrm{C}$, respectively.

To estimate the mass and density of the CO clouds, first we determine the $\mathrm{H}_{2}$ column density from the integrated line flux $W\left({ }^{12} \mathrm{CO}\right)$ using the empirical relation $N\left(\mathrm{H}_{2}\right)=\mathrm{X}_{\mathrm{CO}} \times$ $\mathrm{W}\left({ }^{12} \mathrm{CO}\right)$, where $X_{\mathrm{CO}}$ is the $\mathrm{CO}$ to $\mathrm{H}_{2}$ conversion factor for the ${ }^{12} \mathrm{CO}(\mathrm{J}=1-0)$ transition. We take the canonical value $X_{C O}=2 \times 10^{20} \mathrm{~cm}^{-2} \mathrm{~K} \mathrm{~km} \mathrm{~s}^{-1}$ (Bolatto et al. 2013). The total mass is then calculated from $M=\mu m_{H} S N\left(\mathrm{H}_{2}\right)$, where $\mu$ is the mean molecular mass $(\mu=2.8$ for a relative helium abundance of $25 \%), m_{H}$ is the mass of the hydrogen atom, and $S$ is the area subtended by the source. The areas of the molecular features were approximated by ellipses. To estimate the cloud volume, we considered an ellipsoid with third axis as the mean value of the ellipse semi-axis. For feature $\mathrm{A}$, we integrated the ${ }^{12} \mathrm{CO}$ emission in the $56-65$ $\mathrm{km} \mathrm{s}^{-1}$ velocity range. We estimated the value $W\left({ }^{12} \mathrm{CO}\right)$ from a region defined by the molecular emission superimposed to the SNR (see panel $b$ ), which was approximated by an ellipse of $\left(\right.$ minor $\times$ major semi-axis) $1^{\prime} .7 \times 5^{\prime} .2$. For feature $B$, we integrated the ${ }^{12} \mathrm{CO}$ emission between 66 and $69 \mathrm{~km} \mathrm{~s}^{-1}$ over an ellipse of $1^{\prime} .9 \times 9^{\prime} .3$ For feature $C$, the integration was made in the $\sim 69-75 \mathrm{~km} \mathrm{~s}^{-1}$ range over an ellipse of $2^{\prime} .5 \times 6^{\prime} .3$. Since the distances are roughly compatible within the errors ( $\sim 25 \%$ for this method, Dubner \& Giacani 2015), for the purpose of calculations, we adopt 3.7 and $8.7 \mathrm{kpc}$ as confident near and far distances of each feature, respectively. The obtained masses and number densities are reported in Table 2 .

It is worth noting that the far distance of the molecular features and the SNR G40.5-0.5 ( 8.7 kpc) is compatible, within the errors, with the distance to the pulsar PSR J1907+0631 ( $8.0 \mathrm{kpc})$ located at the center of the SNR and probably associated with the SNR, according to Lyne et al. (2017). Also, the far distance to G40.5-0.5 falls
Table 2. Physical parameters of the molecular features around the SNR G40.5-0.5

\begin{tabular}{cccc}
\hline \hline Feature & $\begin{array}{c}D \\
(\mathrm{kpc})\end{array}$ & $\begin{array}{c}M \\
\left(\mathrm{M}_{\odot}\right)\end{array}$ & $\begin{array}{c}n \\
\left(\mathrm{~cm}^{-3}\right)\end{array}$ \\
\hline $\mathrm{A}$ & 3.7 & 7200 & 660 \\
& 8.7 & 40000 & 280 \\
\hline $\mathrm{B}$ & 3.7 & 9200 & 260 \\
& 8.7 & 51000 & 110 \\
\hline $\mathrm{C}$ & 3.7 & 1000 & 400 \\
& 8.7 & 55000 & 170 \\
\hline
\end{tabular}

within the distance range estimated through the $\Sigma-\mathrm{D}$ relationship. Based on this fact, we suggest that $\sim 8.7$ is the most probable distance to G40.50.5.

\section{ORIGIN OF THE TEV EMISSION}

To date, the association of VER J1907+062 with an offset relic PWN driven by PSR J1907+0602 appears as the most plausible origin for the $\gamma$-ray emission. However, based mainly on the large angular extension and the morphology of the $\mathrm{TeV}$ emission, and its spectral behavior, the contribution of other potential particle accelerators in the field cannot be excluded as well as the possibility that the VHE emission is produced by two object superimposed along the line of sight. In what follow we explore possible scenarios that may give rise to the VHE emission.

As was mentioned in Sect. 1, the SNR G40.5-0.5 lies toward the northern border of the $\mathrm{TeV}$ source and both leptonic and hadronic origin for the VHE $\gamma$-ray production will be considered.

The acceleration of particles (both electrons and protons) at the shock front of a SNR occurs during the remnant's lifetime $t_{s n r}$. In a leptonic scenario, the accelerated electrons interact with a low energy radiation field through the Inverse Compton (IC) scattering, transferring their energy to the seed photons up to TeV energies. To produce detectable $\mathrm{TeV}$ photons, the electrons must have cooled via IC scattering during a time $\tau_{I C} \lesssim t_{s n r}$. The age of G40.5-0.5 was estimated to be between $20-40$ kyr (Downes et al. 1980), but if the remnant is associated with the recently discovered PSR J1907+0631, G40.5-0.5 must have the pulsar's age ( $11 \mathrm{kyr})$. In order to consider all the possible ages quoted for G40.5-0.5, we adopt 11 and $40 \mathrm{kyr}$ as a lower an upper limits for $t_{s n r}$, respectively. Following Kargaltsev et al. (2013), $\tau_{I C} \sim(1+$ $\left.0.144 B_{\mu \mathrm{G}}^{2}\right)^{-1}\left(E_{I C} / \mathrm{TeV}\right)^{-1 / 2}$, where $B_{\mu \mathrm{G}}$ is the magnetic field in $\mu \mathrm{G}$ and $E_{I C}$ is the energy of the $\mathrm{TeV}$ photons. Taking a typical $B=5 \mu \mathrm{G}$, we obtain that in the lifetime of G40.5-0.5 only photons with energies $E_{I C} \gtrsim 4 \mathrm{TeV}$ (for $t_{s n r}=11 \mathrm{kyr}$ ) and $\gtrsim 0.3 \mathrm{TeV}$ (for $t_{s n r}=40 \mathrm{kyr}$ ) can be produced through the IC scattering process. These energies are compatible with the $\gamma$-ray emission from VER $\mathrm{J} 1907+062$, which is detected in the $\sim 0.3-62 \mathrm{TeV}$ energy range (H. E. S. S. Collaboration et al. 2018). We estimate the energy $E_{e}$ of the electrons involved in the IC mechanism from $E_{I C} \sim 4\left(E_{e} / \mathrm{TeV}\right)^{2} \epsilon_{\mathrm{eV}} \mathrm{TeV}$, where $\epsilon_{e V}=\epsilon /(1 \mathrm{eV})$ and $\epsilon$ is the energy of the seed photons. If the CMB is the main 


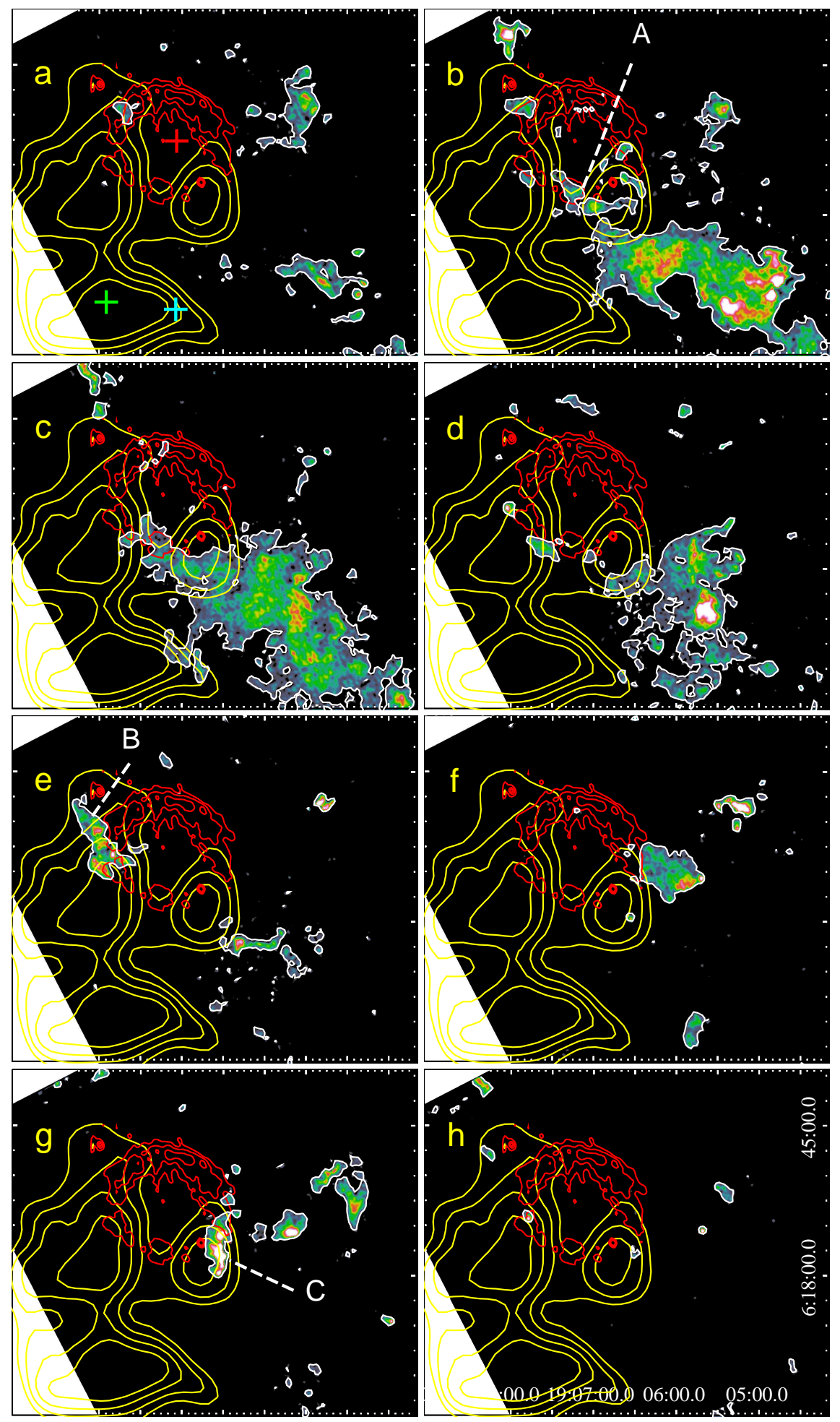

Figure 3. ${ }^{12} \mathrm{CO}(\mathrm{J}=1-0)$ emission toward the SNR G40.5-0.5 integrated over $\sim 2.6 \mathrm{~km} \mathrm{~s}^{-1}$ velocity intervals. The velocity coverage of each panel is: a) $53.1-55.7 \mathrm{~km} \mathrm{~s}^{-1}$, b) $56.3-58.9 \mathrm{~km} \mathrm{~s}^{-1}$, c) $59.6-62.2 \mathrm{~km} \mathrm{~s}^{-1}$, d) $62.8-65.4 \mathrm{~km} \mathrm{~s}^{-1}$, e) $66.1-68.7 \mathrm{~km} \mathrm{~s} \mathrm{~s}^{-1}$, f) $69.3-71.9$ $\left.\mathrm{km} \mathrm{s}^{-1}, g\right) 72.6-75.2 \mathrm{~km} \mathrm{~s}^{-1}$, and $\left.h\right) 75.8-78.4 \mathrm{~km} \mathrm{~s}^{-1}$. The red contours are the radio continuum emission of the SNR at $1.5 \mathrm{GHz}$ and the yellow contours correspond to $\mathrm{TeV}$ emission from VERITAS. The white contour of the ${ }^{12} \mathrm{CO}$ is the same contour level in all the panels. The crosses (shown only in panel a) indicate the positions of the pulsars PSR J1907+0602 (green), PSR J1905+0600 (cyan), and PSR J1907+0631 (red). We label with white letters the molecular features described in the text. The coordinates are equatorial and are shown in panel $h$. 
source of these low energy photons with a temperature $T \sim 3$ $\mathrm{K}\left(\epsilon \sim 3 \times 10^{-4} \mathrm{eV}\right)$, the energy of the electrons is $E_{e} \gtrsim 60$ $\mathrm{TeV}\left(\right.$ for $t_{s n r}=11 \mathrm{kyr}$ ) and $\gtrsim 15 \mathrm{TeV}$ (for $t_{s n r}=40 \mathrm{kyr}$ ).

This simple calculation shows that if the VHE emission around G40.5-0.5 had a leptonic origin, there should be a population of relativistic electrons with energies of several $\mathrm{TeV}$. These high-energy electrons can interact with the ambient magnetic field and produce synchrotron radiation in the keV band. The energy $E_{k e V}$ of the synchrotron photons is obtained from $E_{e} \sim 160 E_{k e V}^{1 / 2} B_{\mu \mathrm{G}}^{-1 / 2} \mathrm{TeV}$ and the corresponding synchrotron cooling time is $\tau_{s y n c} \sim 38 B_{\mu \mathrm{G}}^{-3 / 2} E_{k e V}^{-1 / 2}$ kyr. If $t_{\text {snr }}=11 \mathrm{kyr}$ and taking $B=5 \mu \mathrm{G}$, we get $E_{k e V} \gtrsim 0.6$ $\mathrm{keV}$ and $\tau_{\text {sync }} \lesssim 4.4 \mathrm{kyr}$. If $t_{\text {snr }}=40 \mathrm{kyr}$ and taking $B=5 \mu \mathrm{G}$, we get $E_{k e V} \gtrsim 0.04 \mathrm{keV}$ and $\tau_{\text {sync }} \lesssim 16 \mathrm{kyr}$. Then, electrons accelerated at the shock front have had time enough to cool through synchrotron emission in the lifetime of the SNR and produce X-ray photons which fall within the spectral coverage of current X-ray telescopes. However, XMM-Newton observations failed to detect diffuse emission in the $\mathrm{keV}$ band associated with the SNR (Pandel 2015). We note that the population of Galactic shell type SNR dominated by non-thermal X-ray emission is formed by a reduced number of young sources (typically $\lesssim 2 \mathrm{kyr}$, see the aforementioned SNRcat) that present similar shell-like morphologies in the radio, X-ray and $\mathrm{TeV}$ bands. Representative examples of these sources are SN 1006 (Rothenflug et al. 2004), G347.3-0.5 (Cassam-Chenaï et al. 2004), Vela Jr (Iyudin et al. 2005), and RCW 86 (Vink et al. 2006). In all of them, the VHE emission is attributed mostly to a leptonic mechanism caused by electron acceleration at the shock front of the SNR, with the caveat that even in these sources there are hints of hadronic emission (Miceli et al. 2014; Fukui et al. 2017; Celli et al. 2019). Clearly, the SNR G40.5-0.5 does not match the characteristics of these sources. On the one hand, even if its age is still not firmly establish, all the age estimations show that it is older than 11 kyr. On the other hand, it lacks X-ray emission and the VHE emission does not match the shell in the radio band. Thus, we disfavor a leptonic origin to explain the bulk of the VHE radiation.

Now we analyze the hadronic mechanism, which provides an alternative explanation for VHE $\gamma$-ray emission associated with SNRs. In this scenario, protons accelerated at the shock front collide with target protons of the surrounding ISM and produce $\mathrm{TeV}$ photons via neutral pions decay. Our analysis of the molecular gas around G40.5-0.5 revealed the presence of several clouds in good morphological correspondence with the SNR, favoring a physical connection between them. We can estimate the required density matter $n_{0}$ in the SNR/ISM interaction region to produce the observed $\gamma$-ray flux of VER J1907+062 via the hadronic mechanism. Following Torres et al. (2003), the flux of $\gamma$-ray photons with energies greater than $E$ is given by $F_{\gamma}(>E)=10^{-10} f_{\Gamma} E_{T e V}^{-\Gamma+1} A$, where $f_{\Gamma}$ is a factor depending on the spectral index $\Gamma$, $E_{\mathrm{TeV}}=E / \mathrm{TeV}$, and $A=\theta E_{51} D_{k p c}^{2} n_{0}$ ph $\mathrm{cm}^{-2} \mathrm{~s}^{-1}$, with $\theta$ the fraction of the SN energy that is converted into cosmic ray energy, $E_{51}$ the mechanical energy released by the SN in units of $10^{51} \mathrm{erg}, D_{k p c}$ is the distance in kpc, and $n_{0}$ the ambient density of the ISM in $\mathrm{cm}^{-3}$. The spectral fitting of the TeV emission from VER J1907+062 gives $\Gamma \sim 2.3$ (for which $\left.f_{\Gamma}=0.19\right)$ and $F_{\gamma}(>1 \mathrm{TeV}) \sim 8.4 \times 10^{-12} \mathrm{ph} \mathrm{cm}^{-2}$ $\mathrm{s}^{-1}$ (H. E. S. S. Collaboration et al. 2018). We note that this value corresponds to the $\mathrm{TeV}$ flux of the entire source, so this is an upper limit to the expected $\mathrm{TeV}$ flux produced by the SNR/ISM interaction. Assuming a canonical SN explosion $\left(E_{51}=1\right)$ and $\theta=0.3$, if the SNR is located at the near distance of $\sim 3.7 \mathrm{kpc}$, an ambient density $\gtrsim 20 \mathrm{~cm}^{-3}$ is sufficient to produce the VHE $\gamma$-ray emission of VER J1907+062. If the SNR is at the far distance of $\sim 8.7 \mathrm{kpc}$, the required ambient density is $\gtrsim 120 \mathrm{~cm}^{-3}$. Taking into account that the error in the cloud's densities reported in Table 2 can be of about $40 \%$, we conclude that feature A, which appears over the southern border of the remnant, is dense enough to generate the VHE emission hadronically, independently of whether the SNR and the CO material are either at the near or at the far distance. The densities of feature B and C (located over the eastern and western borders of G40.5-0.5, respectively), are sufficient to produce $\mathrm{TeV}$ photons hadronically only if the SNR and the clouds are located at the near distance of $\sim 3.7 \mathrm{kpc}$. Despite this, the fact that the $\mathrm{TeV}$ source extends far beyond the distribution of the target material suggests that the hadronic scenario probably is not enough to explain the entire VHE emission.

Another possible source of $\mathrm{TeV}$ emission in the field is the pulsar PSR J1907+0631. It is seen in projection close to the center of G40.5-05 and near the northern border of VER J1907+062 but outside the $\gamma$-ray emission region. The distance to the pulsar was estimated to be $\sim 8 \mathrm{kpc}$ in good agreement with the far distance of the remnant. Regardless of the physical connection between both objects, PSR J1907+0631 has a high spin-down flux $\dot{E} / D^{2}$ of $\sim 7.8 \times 10^{33}$ $\mathrm{erg} \mathrm{s}^{-1} \mathrm{kpc}^{-2}$ for a distance $D=8 \mathrm{kpc}$. The TeV flux of VER J1907+062 in the $1-10 \mathrm{TeV}$ band is $\sim 2.3 \times 10^{32} \mathrm{erg}$ $\mathrm{s}^{-1} \mathrm{kpc}^{-2}$, requiring a conversion efficiency from rotational energy of the pulsar to $\gamma$-rays of about 3\%. This is in the range of the expected conversion efficiency of known $\mathrm{TeV}$ PWNe ( $\leq 10 \%$, Gallant 2007), so the pulsar is energetic enough to power the entire $\mathrm{TeV}$ source. However, its location in a region lacking $\gamma$-ray emission and its significant offset from the centroid of VER J1907+062 make it an unlikely candidate as a counterpart of the VHE source. Even more, in the case that PSR J1907+062 is not associated with the SNR G40.5-0.5 and supposing that it was born at the centroid of the $\gamma$-ray emission and traveled for $11 \mathrm{kyr}$ to its actual position leaving a relic $\mathrm{TeV}$ nebula behind, it would require a transverse velocity of $\sim 3800 \mathrm{~km} \mathrm{~s}^{-1}$ (for distance of $8 \mathrm{kpc}$ ). This is an unrealistic fast motion when compared to the velocity of Galactic pulsars (Holland-Ashford et al. 2017; Verbunt et al. 2017).

Regarding PSR J1905+0600, it is an old pulsar (6 Myr) located near the SW border of VER J1907+062. It has a spin-down flux of about $1.6 \times 10^{30} \mathrm{erg} \mathrm{s}^{-1} \mathrm{kpc}^{-2}$ for the estimated distance of $\sim 18 \mathrm{kpc}$, which is several orders of magnitude lower than the TeV flux of VER J1907+062. Therefore it does not fulfill with the energetic requirements to power the whole VHE source.

To summarize, not a single leptonic or hadronic mechanism can fully explain the VHE from VER J1907+062. The most conspicuous sources of $\mathrm{TeV}$ radiation found in the field, namely the pulsar PSR J1907+0602 and the SNR G40.5-0.5 could account for at least a fraction of the VHE emission. If the remnant is located at the far distance of $\sim 8.7 \mathrm{kpc}$, its distance differs considerably from the distance of PSR 
$\mathrm{J} 1907+0602(\sim 3.2 \mathrm{kpc})$. In this case, we propose a scenario in which the $\mathrm{TeV}$ emission is produced by two separated $\gamma$-ray sources. One of them is a PWN powered by PSR J1907+0602 and located at $~ 3.2 \mathrm{kpc}$. Since the pulsar lies over the southern maximum of the $\mathrm{TeV}$ source, it would be powering the $\gamma$-ray emission from the southern hemisphere of VER J1907+062 through the leptonic mechanism. The other $\gamma$-ray source, corresponding to the northern hemisphere of VER J1907+062, would be produced by the interaction between the shock front of the SNR G40.5-0.5 with molecular material via the decay of neutral pions through an hadronic mechanism. This VHE source would be located $\sim 8.7 \mathrm{kpc}$ away.

As suggested in Sect. 3.2, the most probable distance to G40.5-0.5 is $~ 8.7 \mathrm{kpc}$, but we can not completely rule out the possibility that this SNR is located at the near distance of $\sim 3.7 \mathrm{kpc}$. If we consider the errors involved in the estimations, this distance is compatible with the distance of PSR J1907+0602. Also in this case, VER J1907+062 could be the superposition in the line of sight of two nearby, but still separated, $\gamma$-ray sources associated with PSR J1907+0602 and with the SNR G40.5-0.5. Alternatively, if the pulsar and the SNR are at the same distance, VER J1907+062 could be a single $\gamma$-ray source whose emission is powered by the combination of two mechanisms: a leptonic one, associated with a TeV PWN driven by PSR J1907+0602, and a hadronic one, associated with the interaction between the SNR G40.5-0.5 and the surrounding molecular gas.

An improved statistic in the $\mathrm{TeV}$ band is necessary to confirm whether VER J1907+062 is the superposition in the line of sight of two distinct $\gamma$-ray sources powered by different emission mechanisms and located at different distances, or whether it its a single source whose VHE emission is produced by two particle accelerators (the pulsar and the SNR) located at the same distance. The unambiguous determination of the distance of the SNR G40.5-0.5 could shed light on which of the proposed scenarios is taking place.

\section{SUMMARY}

In this paper we present new high-quality radio images of a large region containing the extended $\mathrm{TeV}$ source VER $\mathrm{J} 1907+062$ at $1.5 \mathrm{GHz}$ and a region toward the PSR $\mathrm{J} 1907+0602$ at $6 \mathrm{GHz}$, in both cases with data obtained using the VLA in its D configuration. In spite of the quality of our images, no nebular radio emission has been found toward PSR J1907+0602 (which presents hints of an X-ray PWN) neither toward the other two pulsars in the region, PSR J1907+0631 and PSR J1905+0600. Besides, there is no indication of the existence of a host SNR associated with the mentioned pulsars. From the analysis of the spatial distribution of the ${ }^{12} \mathrm{CO}$ in the vicinity of the SNR G40.5-0.5, we found molecular clouds which match the eastern, southern, and western borders of the remnant and partially overlap peaks of the TeV emission from VER J1907+062. The distance to these clouds (and hence to the SNR) was constraint to be either $\sim 3.7$ or $\sim 8.7 \mathrm{kpc}$. Based on the distance to the pulsar PSR J1907+0631 and its location at the center of the SNR G50.5-0.5 we suggest the far distance as the most probable to the remnant. We discuss possible origins to explain the nature of VER J1907+062 and favor a sce- nario in which the $\mathrm{TeV}$ source consists of two separate $\gamma$-ray sources. One of them of leptonic origin driven by the pulsar PSR J1907+0602 located at $~ 3.2 \mathrm{kpc}$, and the other source of hadronic origin through collisions of ions accelerated at the shock front of the SNR G40.5-0.5 with the molecular gas in its surroundings at a distance $\sim 8.7 \mathrm{kpc}$.

\section{ACKNOWLEDGEMENTS}

The authors wish to thank the anonymous referee since his/her comments improved our manuscript. L.D. is doctoral fellow of CONICET, Argentina. A.P. and E.G. are members of the Carrera del Investigador Científico of CONICET, Argentina. This work was partially supported by Argentina grants awarded by UBA (UBACyT) and ANPCYT. This publication makes use of data from FUGIN, FOREST Unbiased Galactic plane Imaging survey with the Nobeyama 45-m telescope, a legacy project in the Nobeyama 45-m radio telescope.

\section{REFERENCES}

Abdo A. A., et al., 2007, ApJ, 664, L91

Abdo A. A., et al., 2009, Science, 325, 840

Abdo A. A., et al., 2010, ApJ, 711, 64

Aharonian F., et al., 2009, A\&A, 499, 723

Aliu E., et al., 2014, ApJ, 787, 166

Bolatto A. D., Wolfire M., Leroy A. K., 2013, ARA\&A, 51, 207

Cassam-Chenaï G., Decourchelle A., Ballet J., Sauvageot J. L., Dubner G., Giacani E., 2004, A\&A, 427, 199

Castelletti G., Giacani E., Petriella A., 2016, A\&A, 587, A71

Celli S., Morlino G., Gabici S., Aharonian F. A., 2019, MNRAS, 487,3199

Cordes J. M., Lazio T. J. W., 2002, arXiv e-prints, pp astro-ph/0207156

Downes A. J. B., Pauls T., Salter C. J., 1980, A\&A, 92, 47

Dubner G., Giacani 2015, Astronomy and Astrophysics Review, 23, 3

Ferrand G., Safi-Harb S., 2012, Advances in Space Research, 49, 1313

Frail D. A., Scharringhausen B. R., 1997, ApJ, 480, 364

Fukui Y., et al., 2017, ApJ, 850, 71

Gaensler B. M., Slane P. O., 2006, ARA\&A, 44, 17

Gaensler B. M., Stappers B. W., Frail D. A., Moffett D. A., Johnston S., Chatterjee S., 2000, MNRAS, 318, 58

Gallant Y. A., 2007, Ap\&SS, 309, 197

Giacani E., Rovero A. C., Cillis A., Pichel A., Dubner G., 2014, arXiv e-prints, p. arXiv:1412.1673

H. E. S. S. Collaboration et al., 2018, A\&A, 612, A1

Hobbs G., et al., 2004, MNRAS, 352, 1439

Holland-Ashford T., Lopez L. A., Auchettl K., Temim T., Ramirez-Ruiz E., 2017, ApJ, 844, 84

Iyudin A. F., Aschenbach B., Becker W., Dennerl K., Haberl F., 2005, A\&A, 429, 225

Kargaltsev O., Rangelov B., Pavlov G. G., 2013, preprint, (arXiv:1305.2552)

Lyne A. G., et al., 2017, ApJ, 834, 137

Miceli M., Acero F., Dubner G., Decourchelle A., Orlando S., Bocchino F., 2014, ApJ, 782, L33

Pandel D., 2015, in 34th International Cosmic Ray Conference (ICRC2015). p. 743 (arXiv:1512.08140)

Pandel D., Scott R., 2012, in Aharonian F. A., Hofmann W., Rieger F. M., eds, American Institute of Physics Conference 
Series Vol. 1505, American Institute of Physics Conference Series. pp 329-332 (arXiv: 1305.1959), doi:10.1063/1.4772264 Persic M., Salucci P., Stel F., 1996, Monthly Notices of the Royal Astronomical Society, 281, 27 Ranasinghe S., Leahy D. A., 2017, ApJ, 843, 119

Ranasinghe S., Leahy D. A., 2018, AJ, 155, 204

Reid M. J., et al., 2014, The Astrophysical Journal, 783, 130

Rothenflug R., Ballet J., Dubner G., Giacani E., Decourchelle A., Ferrando P., 2004, A\&A, 425, 121

Stil J. M., et al., 2006, AJ, 132, 1158

Sun X. H., Reich P., Reich W., Xiao L., Gao X. Y., Han J. L., 2011, A\&A, 536, A83

Sushch I., Oya I., Schwanke U., Johnston S., Dalton M. L., 2017, A\&A, 605, A115

Torres D. F., Romero G. E., Dame T. M., Combi J. A., Butt Y. M., 2003, Phys. Rep., 382, 303

Umemoto T., et al., 2017, PASJ, 69, 78

Verbunt F., Igoshev A., Cator E., 2017, A\&A, 608, A57

Vink J., Bleeker J., van der Heyden K., Bykov A., Bamba A., Yamazaki R., 2006, ApJ, 648, L33

This paper has been typeset from a $\mathrm{T}_{\mathrm{E}} \mathrm{X} / \mathrm{LAT}_{\mathrm{E}} \mathrm{X}$ file prepared by the author. 